\title{
Produção científica da enfermagem sobre o cuidado familiar de idosos dependentes no domicílio
}

\author{
Scientific production of nursing on the family care of dependent elderly in \\ the household
}

Larissa de Carli Coppetti ${ }^{1}$, Nara Marilene Oliveira Girardon-Perlini ${ }^{1}$, Rafaela Andolhe ${ }^{1}$, Angélica Dalmolin ${ }^{1}$

${ }^{1}$ Programa de Pós-Graduação em Enfermagem, Universidade Federal de Santa Maria (UFSM) - Santa Maria (RS), Brasil.

DOI: https://dx.doi.org/10.7322/abcshs.v44i1.1119

\section{RESUMO}

Este estudo objetiva analisar as tendências das pesquisas na área da enfermagem que abordem a temática do cuidado familiar de idosos dependentes por doenças crônicas no domicílio. Tratase de uma revisão narrativa da literatura, a partir da questão norteadora "Quais as tendências das produções da enfermagem brasileira acerca do cuidado familiar de idosos dependentes por doenças crônicas no domicílio?". A busca foi realizada no mês de agosto de 2017 no Banco de Teses e Dissertações da Associação Brasileira de Enfermagem, acessando os catálogos anuais disponíveis, dos anos de 1998 a 2014. Utilizou-se como estratégias de busca os índices por assunto "cuidadores", "assistência domiciliar" e "pacientes domiciliares". O número total de estudos encontrados foi 233 resumos, que após aplicação dos critérios de inclusão e exclusão, restaram 22 que compuseram o "corpus" deste estudo. A exploração dos dados foi realizada qualitativamente pela análise de conteúdo proposta por Bardin. Destes, 14 são dissertações e oito teses, distribuídas em dez universidades brasileiras, com destaque para região Sudeste. A maioria dos estudos utilizou a abordagem qualitativa, tendo destaque no ano de 2010 com maior concentração de defesas. Conclui-se que o foco principal dos estudos foi o cuidador familiar, desvelando aspectos relacionados à sua caracterização, experiências, vivências, qualidade de vida, sobrecarga, sentido do ser cuidador e suporte social. A compreensão dos aspectos envolvidos no cuidado familiar ao idoso dependente contribui para subsidiar as ações das equipes de saúde, principalmente no preparo/acompanhamento da família que virá ser cuidadora, identificando suas principais dificuldades, necessidades e condições apresentadas.

Palavras-chave: cuidadores; enfermagem domiciliar; doença crônica; revisão.

\begin{abstract}
This study aims to analyze trends in nursing research addressing the issue of family care at home for dependent elderly people because of chronic diseases. This is a narrative review of the literature, based on a guiding question: "What are the trends of Brazilian nursing productions about the family care at home for dependent elderly people because of chronic diseases?". The search was performed in August 2017 at the bank of theses and dissertations of the Brazilian Nursing Association, accessing the available annual catalogs, from 1998 to 2014. Indexes by subject "caregivers" were used as search strategies, "Home care" and "home patients". The total number of studies found was 233 abstracts, after applying inclusion and exclusion criteria, 22 composed the "corpus" of this study. Data extraction was performed qualitatively by the content analysis proposed by Bardin. Of these, 14 are dissertations and eight theses, distributed in ten Brazilian universities mostly in the Southeast region. Most of the studies used the qualitative approach, being highlighted in 2010 with a higher concentration of defenses. It is concluded that the main focus of the studies was the family caregiver, revealing aspects related to their characterization, experiences, life experiences, quality of life, overload, sense of being a caregiver and social support. The understanding of the aspects involved in family care for the dependent elderly contributes to subsidize the actions of the health teams, mainly in the preparation/monitoring of the family that will be caregiver, identifying their main difficulties, needs and conditions presented.
\end{abstract}

Keywords: caregivers; home health nursing; chronic disease; review.

Recebido em: 15/02/2018

Revisado em: 25/04/2018

Aprovado em: 28/06/2018

Autor para correspondência: Larissa de Carli Coppetti - Centro de Ciências da Saúde - Avenida Roraima, 1000, Prédio 26, Sala 1339, Cidade Universitária Bairro Camobi - CEP: 97105-900 - Santa Maria (RS), Brasil - Email: lari_decarli@hotmail.com

Conflito de interesses: nada a declarar. 


\section{INTRODUÇÃO}

O envelhecimento populacional, antes considerado apenas um fenômeno do futuro, hoje se insere na realidade de grande parte da sociedade. Atualmente, estima-se que no Brasil existam cerca de 17,6 milhões de idosos e projeta-se que em 2050, cerca de dois bilhões de pessoas tenham 60 anos ou mais no mundo, concentradas principalmente nos países em desenvolvimento ${ }^{1}$.

O envelhecimento caracteriza-se como um processo natural no qual a reserva funcional dos indivíduos diminui progressivamente, o que, em condições normais, ocorre sem ocasionar problemas. Porém, quando associado a alguma doença pode ocasionar uma condição patológica e, muitas vezes, suscitar dependência de assistência familiar para atividades cotidianas ${ }^{1}$.

À medida que a população envelhece, os fatores de risco para as doenças crônicas não transmissíveis (DCNTs) se acentuam, sendo estas responsáveis por elevados índices de morbimortalidade e comprometimento da independência do idoso. Estas mudanças provenientes das transições demográficas apontam para a necessidade de reformulação do modelo de atenção à saúde, com vistas a prestar atendimento adequado a crescente demanda da população que envelhece. Ademais, intensificam a importância de elaborar e desenvolver ações e políticas públicas capazes de promover o cuidado e a qualidade de vida a esta população $0^{2,3,4}$.

Muitos idosos acometidos por doenças e agravos crônicos demandam acompanhamento constante, uma vez que essas condições tendem a manifestar-se de forma expressiva podendo gerar um processo incapacitante, afetar a funcionalidade da pessoa e dificultar o desempenho das suas atividades de vida diária de forma independente, necessitando a presença de um cuidador familiar ${ }^{1}$.

Esse cuidador pode ser caracterizado como aquele que promove o cuidado dos indivíduos dependentes, de acordo com as necessidades advindas da incapacidade funcional, seja ela temporária ou permanente ${ }^{5}$. Essas ações envolvem suporte físico, atendimento a demandas relacionadas à alimentação, higiene e segurança, além de suporte emocional, sendo estes desenvolvidos de maneira direta e contínua. Os cuidados e as responsabilidades tendem a aumentar conforme a evolução da doença e o grau de dependência apresentado ${ }^{4}$.

As atividades de cuidado no domicilio estão interligadas aos aspectos referentes à estrutura familiar, infraestrutura do domicilio e assistência oferecida pelos serviços de saúde ${ }^{2}$. Cuidar de um idoso dependente pode trazer várias consequências, e variar de aspectos positivos a aspectos negativos, que podem respectivamente, minimizar ou maximizar os sentimentos de sobrecarga e o desconforto emocional do cuidador. A compreensão dessa vivência pode estimular a equipe de saúde, em especial a atenção básica e saúde da família, a obter ações resolutivas nos atendimentos domiciliares ao idoso dependente ${ }^{6}$.

A motivação para a realização deste estudo partiu da necessidade de compreensão ampliada sobre essa temática, uma vez que o idoso dependente por condição crônica carece de um cuidado humanizado e qualificado capaz de suprir suas necessidades. Ademais, o cuidador familiar deve estar amparado pelos serviços de saúde pública, visando receber o apoio e o suporte para as demandas assistenciais no domicílio.

O conhecimento das produções brasileiras na área da enfermagem que abordam o cuidado realizado pelo familiar aos idosos dependentes por doenças crônicas no domicílio, possibilitará a identificação de lacunas na temática, e a partir disto possibilitar o desenvolvimento de estudos futuros que abordem este tema.

Diante disto, o objetivo deste estudo foi analisar as tendências das pesquisas na área da enfermagem que abordem a temática do cuidado familiar de idosos dependentes por doenças crônicas no domicilio.

\section{MÉTODOS}

Trata-se de uma revisão da literatura, do tipo narrativa. Caracteriza-se por sua abordagem ampla, permitindo a descrição de estudos e a imersão do autor sobre determinada temática, bem como domínio acerca do objeto de estudo. Além disso, oportuniza a caracterização das produções sem exigir critérios específicos para sua realização, viabilizando a compilação das produções com a finalidade de analisar os achados e interpretá-los?

Para orientar a pesquisa formulou-se a seguinte questão norteadora: "Quais as tendências das produções da enfermagem brasileira acerca do cuidado familiar de idosos dependentes por doenças crônicas no domicílio?”.

A busca foi realizada no mês de agosto de 2017 no Banco de Teses e Dissertações da Associação Brasileira de Enfermagem (ABEn) - Centro de Estudos e Pesquisas em Enfermagem (CEPEn), acessando os catálogos anuais, utilizou-se como estratégias de busca os índices por assunto "cuidadores", "assistência domiciliar" e "pacientes domiciliares". No intuito de reunir o maior número possível de dissertações e teses, optou-se por não utilizar recorte temporal para inclusão/exclusão na revisão, acessando todos os catálogos disponíveis no banco de teses e dissertações, do ano de 1998 a 2014.

Dentre as estratégias utilizadas, o assunto "cuidadores" resultou em 168 resumos, "assistência domiciliar" em 94 resumos e "paciente domiciliar" em 9 resumos, totalizando 271 estudos. Destes, 38 estavam elencados em mais de um assunto pesquisado, sendo incluídos e analisados uma única vez. Com isso, o número total encontrado foi de 233 resumos.

Após a leitura dos títulos e resumos, foram excluídos 211 estudos, pois não convergiam com a temática proposta, como estudos com crianças, adolescentes e adultos, pacientes não dependentes, com transtornos psiquiátricos, não acometidos por doenças crônicas, cuidados sendo realizados por profissionais, ou cuidados realizados no ambiente hospitalar. Também foram retiradas 
pesquisas metodológicas, resultando assim em 22 resumos que constituíram o corpus da pesquisa.

O processo de identificação, seleção, elegibilidade e inclusão dos estudos, podem ser observados na Figura 1.

Para a organização e posterior análise dos dados, foi elaborado um quadro sinóptico contando as variáveis: título, autor, sujeitos, objetivo, abordagem, tipo de publicação, instituição, estado, ano de publicação, resultados e conclusões. A exploração dos dados foi realizada qualitativamente por meio da análise de conteúdo proposta por Bardin, centrada em torno de três polos cronológicos: pré-análise, exploração do material e tratamento e interpretação dos resultados ${ }^{8}$, sendo então agrupados em duas categorias temáticas: experiências e desafios do cuidado familiar: o papel da enfermagem neste contexto; e as diferentes facetas do cuidado a um idoso dependente.

\section{RESULTADOS E DISCUSSÃO}

O cuidado familiar de idosos dependentes por doenças crônicas no domicilio foi estudada em $14(63,7 \%)$ dissertações e oito $(36,3 \%)$ teses, em dez universidades brasileiras. Destaca-se a região Sudeste com 12 produções, seguido da região Nordeste com seis produções e a região Sul com quatro produções.

Quanto ao ano de defesa, constatou-se que estes se estendem do ano de 2000 a 2014, sendo o ano de 2010 com o maior número (18,1\%), seguido do ano de 2007 (13,6\%). Os anos de 2005, 2006, 2009 e 2011 apresentaram 9,1\% cada, e os anos de 2000, 2001, 2002, 2003, 2008, 2012 e 2014 representam 4,6\% cada. Quanto a abordagem metodológica das investigações, 15 (68\%) foram estudos qualitativos, cinco $(22,8 \%)$ quantitativos, e dois $(9,2 \%)$ mistos quantitativo/qualitativo.

A Tabela 1 apresenta uma síntese dos estudos analisados segundo autor, título, tipo de estudo, ano, local de realização, universidade e principais resultados ${ }^{9-30}$.

As categorias temáticas emergentes da análise de conteúdo são apresentadas e discutidas a seguir.

\section{Experiências e desafios do cuidado familiar: o papel da enfermagem neste contexto}

Ao analisar os estudos encontrados, foi possível perceber que a maioria abordou o cuidador familiar como foco do estudo, trazendo aspectos relacionados à sua caracterização $0^{9,11-14,16,23}$ experiências e desafios do cuidado domiciliar ${ }^{15,19,24,26,27,29}$ qualidade de vida $^{16}$ sobrecarga ${ }^{9,12,16}$ e sentido do ser cuidador ${ }^{13,30}$.

Evidenciou-se que a maioria dos cuidadores são do sexo feminino $^{9,11-14,16,23}$, casadas ${ }^{9,11,13,14,16}$ com baixa escolaridade ${ }^{13,23}$ tendo vínculo de esposas ou filhas ${ }^{11,12,14}$ do idoso doente. Esse resultado também pôde ser encontrado em outros estudos, destacando a predominância da inserção da mulher nas demandas de cuidado ${ }^{6,7}$.
Com relação às experiências relatadas pelos cuidadores abordados nos estudos analisados, emergiram aspectos associados à modificação que o ato de cuidar gera no cotidiano da família ${ }^{29}$ tornando este momento permeado de alterações emocionais e sentimentos como tristeza ${ }^{29}$, medo $^{29}$ e dor $^{26}$. Constata-se que os aspectos negativos envolvidos no cotidiano do cuidador familiar compreendem o conjunto de mudanças em suas vidas, iniciadas desde o momento que se revela a necessidade do cuidado até que o processo de reorganização esteja estabelecido ${ }^{6}$.

A família é considerada o locus preferencial do cuidado ${ }^{27}$, portanto esta necessita de reorganização ${ }^{26}$ e reconfiguração das tarefas e papéis dependendo das demandas emergentes da doença crônica e da incapacidade ${ }^{27}$, sendo o cuidado do idoso dependente considerado um processo complexo ${ }^{12}$. Sob essa ótica, considera-se que estes sentimentos e aspectos negativos relacionados ao cuidado, constituem-se como um desafio a ser superado pela unidade familiar, uma vez que as repercussões de um adoecimento crônico interferem de forma significativa em cada membro da família.

Tais repercussões refletem em alterações no cotidiano da família, podendo de forma ambivalente favorecer situações, ora conflituosas ou de aproximação entre os familiares ${ }^{15}$. Ainda assim, pode ocasionar ao cuidador familiar o estresse, a perda do lazer, a sobrecarga de tarefas e problemas financeiros ${ }^{15}$, bem como sentimentos de insatisfação com a vida e expressões de uma condição solitária ao cuidar do idoso dependente ${ }^{19}$. Não obstante, isto pode ser acentuado quando o cuidado é realizado sem o preparo necessário, fato que é comum na experiência dos cuidadores familiares ${ }^{15}$.

$\mathrm{Na}$ avaliação da qualidade de vida dos cuidadores familiares, os escores das relações sociais e físicas foram os mais afetados, sendo o domínio do meio ambiente com o menor

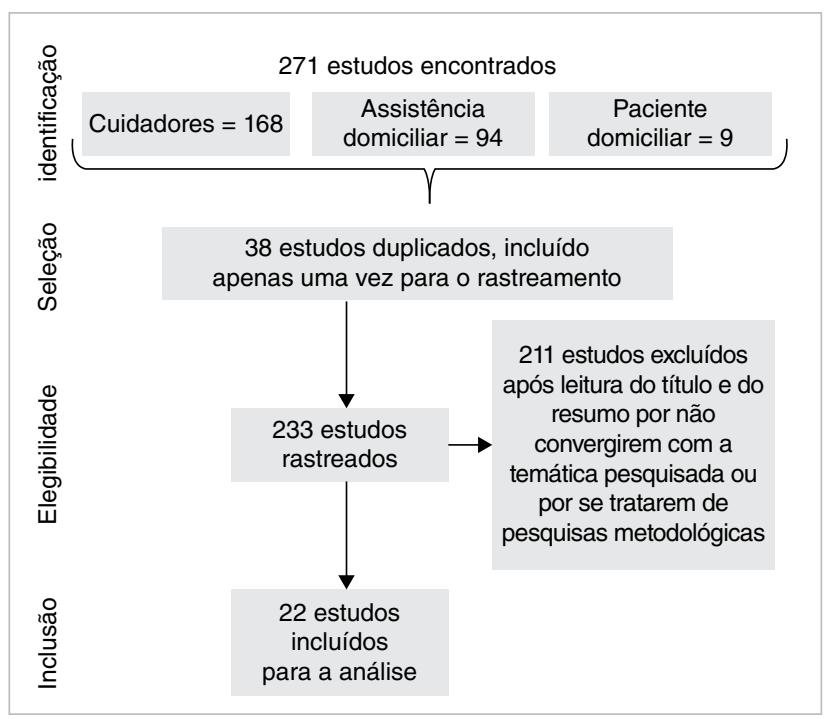

Figura 1: Fluxo de identificação, seleção e inclusão das produções científicas 
Tabela 1: Síntese dos estudos incluídos na revisão segundo autor, título, tipo de estudo, ano, local de realização, universidade e principais resultados.

\begin{tabular}{|c|c|c|c|c|}
\hline Autor/Ano & Título & $\begin{array}{l}\text { Tipo de } \\
\text { estudo }\end{array}$ & Local - Universidade & Principais resultados \\
\hline $\begin{array}{l}\text { Storti \& Marques } \\
2014^{9}\end{array}$ & $\begin{array}{l}\text { Relação entre } \\
\text { sobrecarga do cuidador } \\
\text { familiar e alterações } \\
\text { comportamentais e } \\
\text { funcionais do idoso com } \\
\text { doença de Alzheimer }\end{array}$ & Dissertação & $\begin{array}{l}\text { Ribeirão Preto - } \\
\text { Universidade de São Paulo }\end{array}$ & $\begin{array}{l}\text { Quanto maior a frequência e a gravidade } \\
\text { dos sintomas neuropsiquiátricos, alterações } \\
\text { comportamentais e dependência do idoso maior é a } \\
\text { sobrecarga do cuidador. }\end{array}$ \\
\hline Marins $2012^{10}$ & $\begin{array}{l}\text { Alterações de } \\
\text { comportamento do } \\
\text { idoso com doença de } \\
\text { Alzheimer e o cuidador } \\
\text { informal: Contribuições } \\
\text { para a enfermagem } \\
\text { gerontológica }\end{array}$ & Tese & $\begin{array}{l}\text { Rio de Janeiro - } \\
\text { Universidade Federal do } \\
\text { Rio de Janeiro }\end{array}$ & $\begin{array}{l}\text { Os achados indicam um comprometimento das } \\
\text { ações informativas e educativas de enfermagem ao } \\
\text { cuidador no reconhecimento das ações/alterações de } \\
\text { comportamento do idoso com doença de Alzheimer. }\end{array}$ \\
\hline $\begin{array}{l}\text { Ramos \& } \\
\text { Menezes 201111 }\end{array}$ & $\begin{array}{l}\text { Ritual do cuidar de } \\
\text { idosos com demência } \\
\text { de Alzheimer: história } \\
\text { oral de vida de } \\
\text { cuidadores familiares }\end{array}$ & Dissertação & $\begin{array}{l}\text { Salvador - Universidade } \\
\text { Federal da Bahia }\end{array}$ & $\begin{array}{l}\text { Percebeu-se o ritual de cuidados executado pelos } \\
\text { familiares como um constructo baseado em diferentes } \\
\text { culturas, sendo assim, estes são frutos das culturas e } \\
\text { experiências de cada cuidador. }\end{array}$ \\
\hline Vieira $2011^{12}$ & $\begin{array}{l}\text { Sobrecargas do } \\
\text { cuidador familiar de } \\
\text { idoso com acidente } \\
\text { vascular cerebral } \\
\text { isquêmico e o cuidado } \\
\text { clínico de enfermagem }\end{array}$ & Dissertação & $\begin{array}{l}\text { Fortaleza - Universidade } \\
\text { Estadual do Ceará }\end{array}$ & $\begin{array}{l}\text { A sobrecarga geral esteve entre leve e moderada } \\
\text { em } 92,3 \% \text { dos casos, apresentando associações } \\
\text { estatisticamente significantes com um maior grau } \\
\text { de incapacidade funcional do idoso e com aspectos } \\
\text { relacionados diretamente à vida do cuidador e ao } \\
\text { suporte familiar recebido. }\end{array}$ \\
\hline Gaioli $2010^{13}$ & $\begin{array}{l}\text { Cuidadores de idosos } \\
\text { com doença de } \\
\text { Alzheimer: variáveis } \\
\text { sociodemográficas e } \\
\text { da saúde associadas à } \\
\text { resiliência }\end{array}$ & Tese & $\begin{array}{l}\text { Ribeirão Preto - } \\
\text { Universidade de São Paulo }\end{array}$ & $\begin{array}{l}\text { O cansaço, o esgotamento e o desânimo do cuidador } \\
\text { associaram-se significantemente com alto grau de } \\
\text { resiliência. O estadiamento da demência do idoso } \\
\text { associou-se significantemente com percepção do } \\
\text { estado emocional e da irritabilidade do cuidador. }\end{array}$ \\
\hline Seima $2010^{14}$ & $\begin{array}{l}\text { Relação/Participação no } \\
\text { cuidado entre o cuidador } \\
\text { familiar e o idoso com } \\
\text { Alzheimer }\end{array}$ & Dissertação & $\begin{array}{l}\text { Curitiba - Universidade } \\
\text { Federal do Paraná }\end{array}$ & $\begin{array}{l}\text { A relação/participação entre o familiar e o idoso com } \\
\text { Alzheimer é misteriosa, envolve o amor, a fidelidade } \\
\text { e a presença. Por meio do amor, o cuidador promete } \\
\text { fidelidade e permanece em relação/participação } \\
\text { presente no cuidado ao idoso com Alzheimer. }\end{array}$ \\
\hline $\begin{array}{l}\text { Anjos \& Zago } \\
2010^{15}\end{array}$ & $\begin{array}{l}\text { Repercussões do } \\
\text { cuidar do idoso } \\
\text { em quimioterapia } \\
\text { oncológica na vida do } \\
\text { familiar cuidador }\end{array}$ & Tese & $\begin{array}{l}\text { Ribeirão Preto - } \\
\text { Universidade de São Paulo }\end{array}$ & $\begin{array}{l}\text { Todos os cuidadores relataram falta de preparo para } \\
\text { os cuidados específicos ao idoso em quimioterapia } \\
\text { domiciliar. }\end{array}$ \\
\hline $\begin{array}{l}\text { Santos \& } \\
\text { Tavares } 2010^{16}\end{array}$ & $\begin{array}{l}\text { Qualidade de vida e } \\
\text { sobrecarga e estresse } \\
\text { do cuidador de idoso } \\
\text { com histórico de } \\
\text { acidente vascular } \\
\text { encefálico }\end{array}$ & Dissertação & $\begin{array}{l}\text { Uberaba - Universidade } \\
\text { Federal do Triângulo } \\
\text { Mineiro }\end{array}$ & $\begin{array}{l}\text { A sobrecarga dos cuidadores obteve um escore de } \\
\text { moderada. O maior escore de sobrecarga e estresse } \\
\text { do cuidador foi o preditor de menor escore para o } \\
\text { domínio de qualidade de vida e relações sociais. }\end{array}$ \\
\hline $\begin{array}{l}\text { Santos \& } \\
\text { Menezes } 2009^{17}\end{array}$ & $\begin{array}{l}\text { Relatos orais de } \\
\text { idosos com doença de } \\
\text { Parkinson: concepções } \\
\text { sobre a doença e o } \\
\text { cuidado familiar }\end{array}$ & Dissertação & $\begin{array}{l}\text { Salvador - Universidade } \\
\text { Federal da Bahia }\end{array}$ & $\begin{array}{l}\text { A doença foi percebida como degeneração da } \\
\text { saúde; produção de dependência; limitação do viver } \\
\text { cotidiano; tratamento; constrangimento e instabilidade } \\
\text { emocional. Os significados do cuidado familiar foram } \\
\text { relatados como: satisfação e gratidão; obrigação/ } \\
\text { reciprocidade; segurança no ambiente doméstico; } \\
\text { impaciência; e, suporte físico, emocional e financeiro. }\end{array}$ \\
\hline $\begin{array}{l}\text { Pedreira \& } \\
\text { Lopes } 2009^{18}\end{array}$ & $\begin{array}{l}\text { Modos de ser do } \\
\text { idoso com sequela } \\
\text { de acidente vascular } \\
\text { cerebral: cuidado } \\
\text { familiar }\end{array}$ & Tese & $\begin{array}{l}\text { Salvador - Universidade } \\
\text { Federal da Bahia }\end{array}$ & $\begin{array}{l}\text { Os modos de ser apresentados pelos idosos } \\
\text { dependem da sua temporalidade, influenciada por } \\
\text { fatores como: relações familiares, redes de apoio, } \\
\text { nível sócio econômico e educacional, localização e } \\
\text { extensão do AVC, personalidade entre outros. }\end{array}$ \\
\hline Souza $2008^{19}$ & $\begin{array}{l}\text { Cuidado } \\
\text { transdimensional na } \\
\text { consulta de enfermagem } \\
\text { com cuidadora familiar } \\
\text { de pessoa idosa com } \\
\text { Alzheimer. }\end{array}$ & Tese & $\begin{array}{l}\text { Florianópolis - } \\
\text { Universidade Federal de } \\
\text { Santa Catarina }\end{array}$ & $\begin{array}{l}\text { Durante esse processo de cuidado, foi possível que } \\
\text { as cuidadoras, refletissem sobre o seu modo de } \\
\text { viver, o que influencia de maneira significativa no seu } \\
\text { viver cotidiano, evidenciadas pelas sensações de } \\
\text { bem-estar, alegria, diminuição ou extinção das dores } \\
\text { antes referidas e, por fim, a possibilidade de construir } \\
\text { objetivos para a sua vida. }\end{array}$ \\
\hline
\end{tabular}


Tabela 1: Continuação.

\begin{tabular}{|c|c|c|c|c|}
\hline Autor/Ano & Título & $\begin{array}{l}\text { Tipo de } \\
\text { estudo }\end{array}$ & Local - Universidade & Principais resultados \\
\hline Santana $2007^{20}$ & $\begin{array}{l}\text { Comunicação entre } \\
\text { cuidadores principais e } \\
\text { idosos com demência: } \\
\text { implicações para o } \\
\text { cuidado de enfermagem }\end{array}$ & Tese & $\begin{array}{l}\text { Rio de Janeiro - } \\
\text { Universidade Federal do } \\
\text { Rio de Janeiro }\end{array}$ & $\begin{array}{l}\text { A comunicação entre cuidadores principais e } \\
\text { idosos com demência se dá pela intencionalidade } \\
\text { expressa através dos sentidos do corpo, estando } \\
\text { influenciado pelas características do contexto de } \\
\text { vida dos mesmos. }\end{array}$ \\
\hline Fonseca $2007^{21}$ & $\begin{array}{l}\text { O cuidado domiciliário } \\
\text { ao idoso com doença de } \\
\text { Alzheimer: um enfoque } \\
\text { ao cuidador }\end{array}$ & Dissertação & $\begin{array}{l}\text { Rio de Janeiro - } \\
\text { Universidade Federal do } \\
\text { Estado do Rio de Janeiro }\end{array}$ & $\begin{array}{l}\text { As atividades realizadas pelo cuidador no ambiente } \\
\text { domiciliário envolvem higiene corporal, bucal, } \\
\text { cuidar dos cabelos, alimentação; acompanhamento } \\
\text { e vigilância; necessidades fisiológicas, vestuário } \\
\text { e comunicação. As dificuldades encontradas pelo } \\
\text { cuidador foram delineadas em três aspectos: } \\
\text { Subjetividades do cuidar/cuidado; Família e cuidado; e } \\
\text { Higiene Corporal. }\end{array}$ \\
\hline $\begin{array}{l}\text { Rates \& Sena } \\
2007^{22}\end{array}$ & $\begin{array}{l}\text { Cuidado de saúde do } \\
\text { idoso, no domicílio: } \\
\text { implicações para as } \\
\text { cuidadoras, no Distrito } \\
\text { Ressaca - Município de } \\
\text { Contagem/MG }\end{array}$ & Dissertação & $\begin{array}{l}\text { Belo Horizonte - } \\
\text { Universidade Federal de } \\
\text { Minas Gerais }\end{array}$ & $\begin{array}{l}\text { A dedicação das cuidadoras às tarefas do cuidado } \\
\text { influencia diretamente na qualidade de vida, } \\
\text { ocasionando perdas de oportunidades de trabalho, } \\
\text { de convívio social e lazer, com repercussões na sua } \\
\text { saúde física e emocional. }\end{array}$ \\
\hline $\begin{array}{l}\text { Gratão \& } \\
\text { Rodrigues } \\
2006^{23}\end{array}$ & $\begin{array}{l}\text { Demanda do cuidador } \\
\text { familiar com idoso } \\
\text { demenciado }\end{array}$ & Dissertação & $\begin{array}{l}\text { Ribeirão Preto - } \\
\text { Universidade de São Paulo }\end{array}$ & $\begin{array}{l}\text { Os dados mostram a grande demanda na atividade } \\
\text { de cuidar e conseqüente sobrecarga, revelando a } \\
\text { necessidade urgente de estratégias de suportes } \\
\text { formais e informais para os cuidadores de idosos. }\end{array}$ \\
\hline Vieira $2006^{24}$ & $\begin{array}{l}\text { Sentimentos, saberes } \\
\text { e fazeres do cuidador } \\
\text { principal do idoso com } \\
\text { câncer }\end{array}$ & Dissertação & $\begin{array}{l}\text { Maringá - Universidade } \\
\text { Estadual de Maringá }\end{array}$ & $\begin{array}{l}\text { O estudo revelou que as representações sobre o } \\
\text { câncer estão estreitamente relacionadas com as } \\
\text { práticas terapêuticas domiciliares e os significados } \\
\text { atribuídos ao cuidado, com a percepção das } \\
\text { dificuldades e dos benefícios associados ao papel de } \\
\text { cuidadora principal. }\end{array}$ \\
\hline Pelzer $2005^{25}$ & $\begin{array}{l}\text { Assistência cuidativa } \\
\text { humanística de } \\
\text { enfermagem para } \\
\text { familiares cuidadores de } \\
\text { idosos com doença de } \\
\text { Alzheimer a partir de um } \\
\text { grupo de ajuda mútua. }\end{array}$ & Tese & $\begin{array}{l}\text { Florianópolis - } \\
\text { Universidade Federal de } \\
\text { Santa Catarina }\end{array}$ & $\begin{array}{l}\text { A assistência cuidativa constituiu-se não só } \\
\text { em instância de assistência mais imediata de } \\
\text { vida e saúde, mas também de um espaço de } \\
\text { desenvolvimento pessoal e fortalecimento enquanto } \\
\text { grupo para o encaminhamento de reivindicações } \\
\text { e lutas políticas que façam valer os direitos de } \\
\text { cidadania tanto dos idosos quanto dos seus } \\
\text { cuidadores familiares. }\end{array}$ \\
\hline Messias $2005^{26}$ & $\begin{array}{l}\text { A experiência da } \\
\text { família frente ao idoso } \\
\text { com câncer }\end{array}$ & Dissertação & $\begin{array}{l}\text { Ribeirão Preto - } \\
\text { Universidade de São Paulo }\end{array}$ & $\begin{array}{l}\text { Os sentimentos da família em relação ao } \\
\text { diagnóstico do câncer envolve sofrimento, dor, } \\
\text { revolta diante do impacto do câncer e a aceitação } \\
\text { desta condição por alguns familiares; As cuidadoras } \\
\text { primárias expressaram sua religiosidade perante } \\
\text { a doença; As dificuldades surgidas no contexto } \\
\text { familiar envolve aspectos financeiros e as } \\
\text { mudanças na organização familiar frente ao } \\
\text { processo de cuidar. }\end{array}$ \\
\hline Santos $2003^{27}$ & $\begin{array}{l}\text { Cuidador familiar de } \\
\text { idosos com demências: } \\
\text { um estudo qualitativo } \\
\text { em famílias de origem } \\
\text { nipo-brasileira e } \\
\text { brasileira }\end{array}$ & Tese & $\begin{array}{l}\text { Campinas - Universidade } \\
\text { Estadual de Campinas }\end{array}$ & $\begin{array}{l}\text { A principal diferença observada entre os dois } \\
\text { grupos de famílias não residiu em padrões de } \\
\text { tarefas e de papéis de cuidar, mas nos significados } \\
\text { atribuídos ao apoio familiar, ao cuidado em si } \\
\text { mesmo e ao cuidado e à velhice no contexto do } \\
\text { curso de vida social e individual. }\end{array}$ \\
\hline Lima $2002^{28}$ & $\begin{array}{l}\text { Cuidado domiciliar } \\
\text { ao idoso com doença } \\
\text { de Alzheimer: } \\
\text { representações de } \\
\text { familiares cuidadores, } \\
\text { membros de uma } \\
\text { associação de apoio, na } \\
\text { cidade de Salvador-BA }\end{array}$ & Dissertação & $\begin{array}{l}\text { Salvador - Universidade } \\
\text { Federal da Bahia }\end{array}$ & $\begin{array}{l}\text { A representação gerada foi a de um fenômeno } \\
\text { estressante, mas com possibilidade de produzir } \\
\text { uma maior interação entre os elementos envolvidos, } \\
\text { a depender de alguns fatores como: vínculo afetivo } \\
\text { anterior, tempo de permanência junto ao idoso, } \\
\text { personalidade dos sujeitos, concepção acerca do } \\
\text { envelhecimento, atividade profissional exercida e } \\
\text { história de vida, permitindo uma dimensão valiosa } \\
\text { na constituição das representações sociais do } \\
\text { cuidado domiciliar. }\end{array}$ \\
\hline
\end{tabular}


Tabela 1: Continuação.

\begin{tabular}{|c|c|c|c|c|}
\hline Autor/Ano & Título & $\begin{array}{l}\text { Tipo de } \\
\text { estudo }\end{array}$ & Local - Universidade & Principais resultados \\
\hline Lavinsky $2001^{29}$ & $\begin{array}{l}\text { Cuidar de idosos } \\
\text { com acidente } \\
\text { vascular encefálico: } \\
\text { representações de } \\
\text { familiares cuidadores }\end{array}$ & Dissertação & $\begin{array}{l}\text { Salvador - Universidade } \\
\text { Federal da Bahia }\end{array}$ & $\begin{array}{l}\text { As representações que envolvem o AVE sao tidas como } \\
\text { algo inexplicável, repentino, o fim da vida, deixando } \\
\text { o idoso como criança; a falta de ajuda dificulta o } \\
\text { cuidado, modificando o quotidiano familiar; o cuidar } \\
\text { de idosos, em domicílio, envolve manifestações de } \\
\text { sentimentos e estreita a convivência familiar, apresenta } \\
\text { sentimentos como tristeza, medo, nervoso, satisfação, } \\
\text { pena, carga pesada, amor dos familiares; a orientação } \\
\text { de um profissional contribui para o fortalecimento do } \\
\text { familiar cuidador, expressa dificuldades amenizáveis se } \\
\text { houvesse orientação profissional. }\end{array}$ \\
\hline Caldas $2000^{30}$ & $\begin{array}{l}\text { Sentido do ser cuidando } \\
\text { de uma pessoa idosa } \\
\text { que vivencia um } \\
\text { processo de demência }\end{array}$ & Tese & $\begin{array}{l}\text { Rio de Janeiro - } \\
\text { Universidade Federal do } \\
\text { Rio de Janeiro }\end{array}$ & $\begin{array}{l}\text { Na ótica assistencial, é necessário discutir o tempo } \\
\text { e o cuidado. Em relação ao tempo, o cuidador pode } \\
\text { estar aberto para fazer ajustes no seu cotidiano } \\
\text { e não anular as suas próprias possibilidades. Em } \\
\text { relação ao cuidado, é preciso que os profissionais de } \\
\text { saúde compreendam que no início, os cuidadores até } \\
\text { poderão cumprir as prescrições e orientações, mas } \\
\text { depois ele próprio decidirá, livre e independente dos } \\
\text { profissionais de saúde, a partir das experiências e } \\
\text { conhecimentos adquiridos. }\end{array}$ \\
\hline
\end{tabular}

escore $^{16}$. A sobrecarga dos cuidadores variou de leve/modera$\mathrm{da}^{12,16}$ a pequena ${ }^{9}$, sendo que os maiores níveis de sobrecarga estão relacionados com o grau de incapacidade funcional e dependência do idoso $0^{9,12}$.

Em concordância, um estudo afirma que várias condições envolvidas no cuidado, após uma doença crônica acompanhada da dependência, são condicionantes para o aparecimento da sobrecarga. Nesse contexto, destaca-se a complexidade de cuidados exigidos, o tempo necessário de assistência, bem como as características do cuidador e do ser cuidado envolvidos neste processo ${ }^{31}$.

Quanto ao sentido do ser cuidador, destaca-se o sentimento de retribuição e de satisfação ao cuidar, onde a ajuda recebida de outras pessoas, sejam familiares ou profissionais da saúde, favorece a capacidade resiliente do cuidador ${ }^{13}$. Contrariamente, outro estudo refere que a pessoa que cuida de um familiar dependente sente-se presa, mas cuida porque não vê outra saída, ocupando-se mais do que se preocupando com o ato de cuidar ${ }^{30}$.

A alta hospitalar de um idoso dependente por doença crônica e a necessidade da transferência do cuidado para o ambiente domiciliar, constitui-se um desafio para a família que virá a ser cuidadora, estando relacionado ao exercício do cuidado e o suporte recebido para tal. Alguns estudos analisados versam sobre o conhecimento das práticas exercidas no cuidado domiciliar ${ }^{11,21,23,28}$ e a importância da relação entre a equipe de saúde e cuidadores, com vistas a fornecer suporte social para o cuidado ${ }^{10,11,22,25}$.
O ritual de cuidados aos idosos dependentes é executado como um construto baseado em diferentes culturas ${ }^{11}$. As práticas são as mais variadas, com destaque para atividades diárias de vida, como higiene corporal, alimentação, acompanhamento e vigilância, necessidades fisiológicas, vestuário e comunicação ${ }^{21}$. Sendo estes cuidados são realizados pelos cuidadores de forma ininterrupta ${ }^{11}$, exigem tempo, recursos e paciência ${ }^{28}$, ocasionando grande demanda para o cuidador familiar ${ }^{23}$.

Ainda neste contexto, um dos estudos apreende que cuidar requer entendimento e desenvolvimento de estratégias e habilidades emocionais, uma vez que esta prática se encontra imersa na subjetividade individual de quem necessita de cuidados e do próprio cuidador ${ }^{21}$. Esta afirmação foi evidenciada em demais estudos, confirmando o fato de que assumir as demandas de cuidado no domicilio sem domínio das habilidades e conhecimentos necessários, associada à natureza crônica dos idosos, pode oportunizar o aparecimento de consequências emocionais e físicas de cunho negativo, afetando a qualidade da assistência prestada ${ }^{32}$.

Os resultados dos estudos desvelam que a atenção das equipes de saúde pública aos cuidadores familiares deve buscar atender cada família em particular, respondendo a suas necessidades e desenvolvendo o processo de cuidar junto a cada cuidador ${ }^{25}$. Sendo assim, as ações educativas de enfermagem devem pautar-se no contexto social, cultural e econômico em que estão inseridos, bem como, nas demandas de cuidado trazidas por esse cuidador ${ }^{10}$. 
Com relação aos cuidadores de idosos com demência, um estudo reforça a necessidade de intensificar a assistência, seja prestada pelas equipes ou gestores da área da saúde, com vistas a elaborar e implementar políticas públicas para fornecer suporte à família que virá a ser cuidadora no domicílio ${ }^{11}$.

Diante do exposto, a enfermagem possui papel essencial na promoção da assistência às famílias e aos cuidadores, possibilitando prepará-los de acordo as demandas necessárias para o cuidado no domicílio. Fornecer orientações, suporte e acompanhamento favorece o desenvolvimento de conhecimento e habilidades, capazes de melhorar a qualidade do cuidado prestado e minimizar os aspectos e sentimentos negativos vivenciados pelos cuidadores familiares.

\section{As diferentes facetas do cuidado à um idoso dependente}

Algumas investigações voltaram-se para a compreensão do cuidado no ambiente domiciliar, tendo seus conteúdos direcionados aos aspectos referentes à caracterização e grau de dependência dos idosos ${ }^{9,11-13}$, a relação interpessoal entre cuidadores e idosos $^{14,20}$, alterações comportamentais ${ }^{10}$, modos de ser do idoso ${ }^{18}$ e suas concepções sobre a doença e o cuidado recebido ${ }^{17}$.

Os participantes dos estudos foram cuidadores familiares de idosos dependentes por doenças crônicas, dentre elas: demência sem definição de causa ${ }^{20,23,27,30}$ Alzheimer ${ }^{9-11,13,14,19,21,25,28}$, parkinson $^{17}$, câncer ${ }^{15,24,26}$ e acidente vascular cerebral ${ }^{12,16,18,29}$. Outro estudo apenas caracteriza o idoso como dependente por doença crônica ${ }^{22}$, sem especificar patologia.

Quanto a caracterização dos idosos cuidados, a faixa etária variou de 60 a $96 \operatorname{anos}^{9,13}$, com predomínio do sexo feminino ${ }^{9,11,13}$. Quanto ao grau de dependência, a maioria dos idosos apresentavam incapacidade moderada ${ }^{9,12}$ para as atividades de vida diária.

A relação entre cuidadora e pessoa cuidada possuem alguns aspectos que facilitam e/ou dificultam a execução do cuidado no domicílio. As singularidades e mudanças cognitivas apresentadas pelos idosos em decorrência da patologia são consideradas pelos cuidadores familiares como aspectos dificultadores, enquanto o estímulo para as atividades de autocuidado é visto como condição que facilita o cuidado no domicilio e o relacionamento com o idoso ${ }^{22}$.

A comunicação entre idosos e cuidadores ocorre a partir da intencionalidade expressa por meio dos sentidos do corpo, sendo influenciada pelas características do contexto em que estão inseridos ${ }^{20}$. Esta relação envolve amor, fidelidade e presença, uma vez que, pelos laços de amor, os cuidadores comprometem-se fielmente com sua presença e relação/participação no cuidado ao idoso ${ }^{14}$.
Algumas alterações comportamentais do idoso, como agitação, teimosia, agressividade verbal e irritabilidade, apesar de serem reconhecidas como alterações ocasionadas pela doença, afetam o processo de cuidar, causando interferência e incômodo à vida do cuidador. Com isso, o padrão relacional entre cuidador e idoso passa por algumas alterações, que culminam na necessidade de novas estratégias de manejo a serem incorporadas no dia a dia de cuidado ${ }^{10}$.

Quanto ao modo de ser dos idosos, os estudos indicam que o idoso ao se perceber em situação de dependência, desvela um cotidiano vazio acompanhado da angústia pelo seu ser-no-mundo ${ }^{18}$. Percebe a doença como degeneração do seu estado de saúde, produção da dependência, limitação do viver cotidiano, constrangimento e instabilidade emocional ${ }^{17}$. Esse resultado pôde ser evidenciado em mais estudos, confirmando o sentimento de impotência e inutilidade vivenciado pelo idoso diante do adoecimento e da dependência, favorecendo o seu descontrole emocional e podendo ocasionar conflitos familiares ${ }^{33}$.

Com relação ao cuidado recebido, os idosos atribuem o significado de satisfação e gratidão, suporte físico, emocional e financeiro, segurança pela permanência no ambiente doméstico, mas também vivenciam a impaciência ${ }^{17}$. Contudo, evidencia-se na literatura que existe o reconhecimento e a valorização do cuidado recebido, uma vez que a compreensão, o apoio e a dedicação do familiar são fatores que contribuem para o enfrentamento do idoso na condição dependente ${ }^{33}$.

\section{CONSIDERAÇÕES FINAIS}

Este estudo possibilitou conhecer o conteúdo das teses e dissertações brasileiras desenvolvidas na área da enfermagem, sobre o cuidado do cuidador familiar ao idoso dependente por doença crônica no domicílio. As tendências das produções científicas evidenciam que a maioria das investigações nesta área utilizam a abordagem qualitativa, versando sobre as experiências e desafios enfrentados pelos cuidadores familiares no dia a dia do cuidado domiciliar.

Nesse sentido, pode-se perceber que cuidar no domicílio pode gerar desconforto, medo, susto, readaptações trazendo consigo muitas dificuldades. Constatou-se que alguns cuidadores não receberam orientações/informações referentes aos cuidados, refletindo uma limitação no suporte e no apoio dispensado pelas equipes de saúde à esta população.

Com isto, este estudo traz a tona aspectos que ainda necessitam ser considerados pelos serviços públicos de saúde, contribuindo para uma reflexão que poderá servir de subsidio para redirecionar as ações na assistência e preparo da família que virá a ser cuidadora no domicílio, possibilitando identificar as 
principais dificuldades, necessidades e condições apresentadas, e a partir destas, desenvolver uma rede de suporte comunitário com vistas a atender as demandas percebidas.

As lacunas encontradas no conhecimento produzido, dizem respeito ao desenvolvimento de estudos que abordem as habilidades e as competências prévias aos cuidadores familiares, para assim possibilitar subsídios aos profissionais da área da saúde durante o preparo da família para assumir os cuidados, de acordo com as necessidades de cada uma. Outra lacuna se refere à maneira como ocorre à reorganização familiar para prestar o cuidado domiciliar. Sugere-se assim, a realização de estudos que abordem estas perspectivas, possibilitando a compreensão destas dimensões do processo de cuidar de idosos dependentes no domicílio.

\section{REFERÊNCIAS}

1. Brasil. Ministério da Saúde. Secretaria de Atenção à Saúde. Departamento de Atenção Básica. Envelhecimento e saúde da pessoa idosa. Brasília: Ministério da Saúde; 2007.

2. Brasil. Ministério da Saúde. Secretaria de Atenção à Saúde. Departamento de Atenção Básica. Caderno de atenção domiciliar. V.1. Brasília: Ministério da Saúde, 2012.

3. Melo DS, Martins RD, Jesus RPFS, Samico IC, Santo ACGE. Avaliação da responsividade de um serviço de saúde público sob a perspectiva do usuário idoso. Rev Saúde Pública. 2017;51:62 http://dx.doi.org/10.1590/s1518-8787.2017051006854

4. Lindolpho MC, Caldas CP, Acioli S, Vargens OMC. O cuidador de idoso com demência e a política de atenção à saúde do idoso. Rev Enferm UFPE. 2014; 8(12):4381-90.

http://dx.doi.org/10.5205/reuol.6679-58323-1-ED.0811201425

5. Oliveira DC, D'Elboux MJ. Estudos nacionais sobre cuidadores familiares de idosos: revisão integrativa. Rev Bras Enferm. 2012; 65(5):829-38.

http://dx.doi.org/10.1590/S0034-71672012000500017

6. Couto AM, Castro EAB, Caldas CP. Vivências de ser cuidador familiar de idosos dependentes no ambiente domiciliar. Rev Rene. 2016;17(1):76-85.

http://dx.doi.org/10.15253/rev\%20rene.v17i1.2624

7. Brum CN, Zuge SS, Rangel RF, Freitas HMB, Pieszak GM. Revisão narrativa da literatura: aspectos conceituais e metodológicos na construção do conhecimento da enfermagem. In: Lacerda MR, Costenaro RGS. Metodologias da pesquisa para a enfermagem e saúde. Porto Alegre: Moriá, 2015.

8. Bardin L. Análise de conteúdo. São Paulo. Ed. 70, 2011

9. Storti LB, Marques S. Relação entre sobrecarga do cuidador familiar e alterações comportamentais e funcionais do idoso com doença de Alzheimer. Dissertação (Mestrado) - Universidade de São Paulo. Ribeirão Preto: 2014.

10. Marins AMF. Alterações de comportamento do idoso com doença de Alzheimer e o cuidador informal: contribuições para a enfermagem gerontológica. Tese (Doutorado) - Universidade Federal do Rio de Janeiro. Rio de Janeiro: 2012; p.245.

11. Ramos JLC, Menezes MR. Ritual do cuidar de idosos com demência de Alzheimer: história oral de vida de cuidadores familiares. Dissertação (Mestrado) - Universidade Federal da Bahia. Salvador: 2011

12. Vieira CPB. Sobrecargas do cuidador familiar de idoso com acidente vascular cerebral isquêmico e o cuidado clínico de enfermagem. Dissertação (Mestrado) - Universidade Estadual do Ceará. Fortaleza: 2010.
13. Gaioli CCLO. Cuidadores de idosos com doença de Alzheimer: variáveis sociodemográficas e da saúde associadas à resiliência Tese (Doutorado) - Universidade de São Paulo. Ribeirão Preto: 2010.

14. Seima MD. Relação/Participação no cuidado entre o cuidador familiar e o idoso com Alzheimer. Dissertação (Mestrado) Universidade Federal do Paraná. Curitiba: 2010.

15. Anjos ACY, Zago MMF. As repercussões do cuidar do idoso em quimioterapia oncológica na vida do familiar cuidador. Tese (Doutorado) - Universidade de São Paulo. Ribeirão Preto: 2010.

16. Santos NMF, Tavares DMS. Qualidade de vida e sobrecarga de estresse do cuidador de idoso com histórico de acidente vascular encefálico. Dissertação (Mestrado) - Universidade Federal do Triângulo Mineiro. Uberaba: 2010.

17. Santos ISC, Menezes MR. Relatos orais de idosos com doença de Parkinson: concepções sobre a doença e o cuidado familiar. Dissertação (Mestrado) - Universidade Federal da Bahia. Salvador: 2009

18. Pedreira LC, Lopes RLM. Modos de ser do idoso com sequela de acidente vascular cerebral: cuidado familiar. Tese (Doutorado) Universidade Federal da Bahia. Salvador: 2009.

19. Souza EBM. Cuidado transdimensional na consulta de enfermagem com cuidadora familiar de pessoa idosa com Alzheimer. Dissertação (Mestrado) - Universidade Federal de Santa Catarina. Florianópolis: 2008.

20. Santana RF. Comunicação entre cuidadores principais e idosos com demência: implicações para o cuidado de enfermagem. Tese (Doutorado) - Universidade Federal do Rio de Janeiro. Rio de Janeiro: 2007.

21. Fonseca AM. O cuidado domiciliário ao idoso com doença de Alzheimer: um enfoque ao cuidador. Dissertação (Mestrado) Universidade Federal do Estado do Rio de Janeiro. Rio de Janeiro: 2007.

22. Rates HF, Sena RR. Cuidado de saúde do idoso, no domicílio: implicações para as cuidadoras, no Distrito Ressaca - Município de Contagem/MG. Dissertação (Mestrado) - Universidade Federal de Minas Gerais. Belo Horizonte: 2007.

23. Gratão ACM, Rodrigues RAP. Demanda do cuidador familiar com idoso demenciado. Dissertação (Mestrado) - Universidade de São Paulo. Ribeirão Preto: 2006.

24. Vieira MCU. Sentimentos, saberes e fazeres do cuidador principal do idoso com câncer. Dissertacão (Mestrado) - Universidade Estadual de Maringá. Maringá: 2006. 
25. Pelzer MT. Assistência cuidativa humanística de enfermagem para familiares cuidadores de idosos com doença de Alzheimer a partir de um grupo de ajuda mútua. Tese (Doutorado) - Universidade Federal de Santa Catarina. Florianópolis: 2005.

26. Messias DX. A experiência da família frente ao idoso com câncer. Dissertação (Mestrado) - Universidade de São Paulo. Ribeirão Preto: 2005.

27. Santos SMA. Cuidador familiar de idosos com demências: um estudo qualitativo em famílias de origem nipo-brasileira e brasileira. Tese (Doutorado) - Universidade Estadual de Campinas. Campinas: 2003.

28. Lima CFM. O cuidado domiciliar ao idoso com doença de Alzheimer: representações de familiares cuidadores, membros de uma associação de apoio, na cidade de Salvador-BA. Dissertação (Mestrado) - Universidade Federal da Bahia. Salvador: 2002

29. Lavinsky AE. Cuidar de idosos com acidente vascular encefálico: representações de familiares cuidadores (O).
Dissertação (Mestrado) - Universidade Federal da Bahia. Salvador: 2001

30. Caldas CP. O sentido do ser cuidando de uma pessoa idosa que vivencia um processo de demência. Rev Enferm UERJ 2000;8(1):9-14

31. Mitchell LA, Hirdes J, Poss JW, Boyd CS, Caldarelli H, Martin L. Informal caregivers of clients with neurological conditions: profiles, patterns and risk factors for distress from a home care prevalence study. BMC Health Serv Res. 2015;15:350.

http://dx.doi.org/10.1186/s12913-015-1010-1

32. Landeiro MJL, Peres HHC, Martins T. Avaliação das necessidades informacionais dos cuidadores domiciliares. Rev Enferm UFSM. 2015;5(3):486-9.

http://dx.doi.org/10.5902/2179769216886

33. Tavares KO, Scalco JC, Vieira L, Silva JR, Bastos CCCB Envelhecer, adoecer e tornar-se dependente: a visão do idoso. Rev Kairós Gerontol. 2012;15(3):105-18. 